\title{
含负氢配体的单核钉催化的降冰片烯开环易位聚合催化研究
}

\author{
陈锦森朱聪之陈勋王金虎朱进* \\ (南京大学化学化工学院 微结构国家实验室 配位化学国家重点实验室 高分子科学与工程系 南京 210093)
}

\begin{abstract}
摘要 设计的含负氢配体的单核钓配合物 $\mathrm{Ru}\left(p\right.$-cymene) $\mathrm{HClPCy}_{3}$ 高活性地催化了降冰片烯开环易位聚合反应(ROMP), 并通过设计的 $\mathrm{Ru}(p$-cymene $) \mathrm{H}_{2} \mathrm{PCy}_{3}$ 对降冰片烯的催化活性研究证明了单核催化剂需同时含有氯原子配体，提出了反应 机理.
\end{abstract}

关键词 烯烃开环易位聚合反应; 降冰片烯; 负氢配体

\section{Studies on Ring-Opening Metathesis Polymerization of Norborene Catalyzed by Hydride Containing Mononuclear Ruthenium Complex}

\author{
Chen, Jinsen \\ Zhu, Congzhi \\ Chen, Xun \\ Wang, Jinhu \\ Zhu, Jin*
}

(Department of Polymer Science and Engineering, School of Chemistry and Chemical Engineering, State Key Laboratory of Coordination Chemistry, Nanjing National Laboratory of Microstructures, Nanjing University, Nanjing 210093, China)

\begin{abstract}
Ring-opening metathesis polymerization (ROMP) has emerged as an important tool for the preparation of architecturally unique macromolecules from cyclic olefins. A wide variety of catalytic systems, ranging from simple metal salts to highly sophisticated alkylidene metal complexes, have been used for the achievement of this synthetically useful transformation. Especially, tremendous efforts have been directed toward the ruthenium-based catalytic systems as well as the understanding of their reactivity patterns. And Grubbs catalysts represent the ultimate in terms of activity, but they are not cost effective due to the sophisticated ligand. Therefore, alternative catalyst precursors that are more readily accessible have been and are still being actively developed. Therefore, a hydride ligand containing mononuclear ruthenium complex $\mathrm{Ru}\left(p\right.$-cymene) $\mathrm{HClPCy}_{3}$ reported here was designed to catalyze ring-opening metathesis polymerization of norbornene with high activity. The hydride ligand is critical based on its high trans effect (for ligand dissociation) and the ability to generate carbene species through plausible migratory insertion and $\alpha$-elimination steps. This catalytic system bearing hydride ligand could be an alternative for the well-defined Ru-based initiators which rules out the need of installation of complex ancillary ligands like NHC or O-Ligand in the coordination sphere or the need of activation with diazo compounds in the search for novel active catalytic species. Further study on the catalytic polymerization activity of $\mathrm{Ru}(p$-cymene $) \mathrm{H}_{2} \mathrm{PCy}_{3}$ compared to the $\mathrm{Ru}(p$-cymene $) \mathrm{HClPCy}_{3}$ system revealed that chloride ligand was also crucial to the hydride containing mononuclear ruthenium system, which contains easily dissociated ligand $p$-cymene and large phosphine ligand that stabilizes the metal complex. And the possible mechanism for the reaction was proposed.

Keywords ring-opening metathesis polymerization; norbornene; hydride ligand
\end{abstract}

\section{1 引言}

烯烃开环易位聚合反应(ROMP)是一种极为有效的 具有广泛应用前景的生成高分子聚合物的实验手段. 经 过半个世纪的发展, 以烯烃复分解反应为中心, 发展衍 化出了多种化学反应, 成为了化学合成中一种重要的方 法. 20 世纪 80 年代, 钓配合物催化体系的发现使得大部 分的具有不同官能团的单体可以直接用于烯烃复分解 反应，同时降低了氧气和水对于反应的影响 ${ }^{[1 \sim 4]}$. 其中, 最具有代表性就是 Grubbs 课题组发明的 Grubbs 催化剂, 该类催化剂反应活性好, 官能团容忍性好, 已经发展出
Grubbs 第一代催化剂 ${ }^{[5,6]}$ 、Grubbs 第二代催化剂 ${ }^{[7]}$ 以及 Hoveyda 等研究出的 Hoveyda-Grubbs 催化剂 ${ }^{[8,9]}$. 尽管 如此, 其复杂难合成的配体以及钉金属的价格仍然是其 大规模使用的制约因素 ${ }^{[10 \sim 13]}$. 所以，人们针对可以通过 不同反应机理催化开环易位聚合的催化剂也一直在探 索, 试图得到催化效率更高, 聚合更迅速, 适用单体范 围更广的非 Grubbs 类型的催化体系 ${ }^{[14-21]}$. 比如, 本课 题组在已经报道的工作中设计出 $\left[\mathrm{Ru}(p \text {-cymene }) \mathrm{Cl}_{2}\right]_{2}$ 与 胺硼烷 $\mathrm{BH}_{3} \cdot \mathrm{NMe}_{3}$ 共同作用下的双组分催化体系 $\left[\mathrm{Ru}(p \text {-cymene }) \mathrm{Cl}_{2}\right]_{2} / \mathrm{BH}_{3} \cdot \mathrm{NMe}_{3}$ 以及从该双组分体系分

\footnotetext{
*E-mail: jinz@nju.edu.cn; Tel.: 025-83686291; Fax: 025-83317761

Received October 9, 2014; published October 10, 2014.

Supporting information for this article is available free of charge via the Internet at http://sioc-journal.cn.

Project supported by the National Natural Science Foundation of China (No. 21274058) and the National Basic Research Program of China (No. 2011CB935801).

项目受国家自然科学基金(No. 21274058)和国家重点基础研究发展计划(No. 2011CB935801)资助.
} 
离出的含负氢桥双核配合物的单组分双核体系 $\left[\{\mathrm{Ru}(p \text {-cymene })\}_{2}(\mu-\mathrm{H})(\mu-\mathrm{Cl}) \mathrm{Cl}_{2}\right]$ 都有效地催化了降冰片 烯的烯烃开环聚合反应(Figure 1) ${ }^{[22]}$. 通过合成多种含 负氢配体的双核配合物及对它们聚合活性的研究我们 验证了负氢配体对配合物的聚合催化反应的重要作用, 指出了含双负氢桥的配体物为活性中心的假设并提出 了可能的机理. 将上述的双核金属中心修改为单核金属 中心, 去除金属桥键, 可以简化对催化机理的研究, 因 此本文设计了含负氢配体的单核钉配合物 $\mathrm{Ru}(p$-cymene $) \mathrm{HClPCy}_{3}$ 催化降冰片烯烯烃开环易位聚合 反应, 并对其催化机理进行了研究.
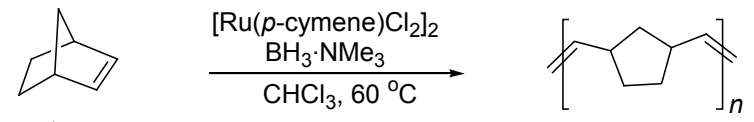<smiles>C1=CC2CCC1C2</smiles>

$\frac{\left[\{\mathrm{Ru}(p \text {-cymene })\}_{2}(\mu-\mathrm{H})(\mu-\mathrm{Cl}) \mathrm{Cl}_{2}\right]}{\mathrm{CHCl}_{3}, 60{ }^{\circ} \mathrm{C}}$

$\left[\{\mathrm{Ru}(p \text {-cymene })\}_{2}(\mu-\mathrm{H})(\mu-\mathrm{Cl}) \mathrm{Cl}_{2}\right]$<smiles>C1=CC2CCC1C2</smiles>

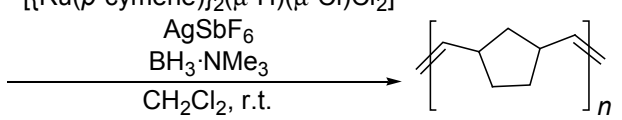

图 1 含负氢配体的双核钓配合物催化的降冰片烯的开环易位聚合 Figure 1 Ring-opening metathesis polymerization of norbornene catalyzed by hydride containing di-nuclear ruthenium complex

\section{2 结果与讨论}

\section{1 单核 $\mathrm{Ru}\left(p\right.$-cymene) $\mathrm{HCIPCy}_{3}$ 催化体系的设计与 反应条件优化}

本课题组在对含负氢配体的双核钉配合物对降冰 片烯的开环易位聚合的研究基础上, 设想如果设计出一 种单核钉配合物作为催化剂时便可以使反应体系得到 简化研究. 本课题组进而认为含有以下三个特征的单核 钓配合物可以催化降冰片烯的 ROMP 反应, 即含有: (1) 易解离的 $p$-cymene 配体, 更好地暴露反应位点促使单 体更好地与催化中心金属配位; (2)负氢配体, 利用 $\mathrm{M}-\mathrm{H}$ 键加成反应更好地引发反应; (3)大位阻的磷配体 $\mathrm{PCy}_{3}$, 稳定单核配合物. 因此, 本文以上述三个特征设 计并以 $\mathrm{Ru}\left(p\right.$-cymene) $\mathrm{HClPCy}_{3}$ 作为催化剂来催化降冰片 烯的 ROMP 反应 ${ }^{[23]}$.

首先, 针对反应溶剂以烯烃开环易位聚合反应中常 用的三种溶剂 $\mathrm{CHCl}_{3}, \mathrm{THF}, \mathrm{PhCl}$ 作为反应溶剂进行篎 选, 结果显示, 在三种溶剂中 $\mathrm{Ru}\left(p\right.$-cymene) $\mathrm{HClPCy}_{3}$ 可 以很好地催化降冰片烯的 ROMP 反应(如 Table 1 所示). 在 $\mathrm{CHCl}_{3}$ 中的空白实验(相同反应条件下去除单核钉配 合物催化剂, Supporting Information)表明 $\mathrm{Ru}(p$-cymene)$\mathrm{HClPCy}_{3}$ 对聚合反应的发生是必需的.

针对反应体系进行优化时, 降冰片烯 ROMP 聚合 反应中单体浓度也是反应的关键参数, 因此, 对单体浓 度的优化结果表明(如 Supporting Information Figure S1 所示), 在 $0.5 \mathrm{~mol} / \mathrm{L}$ 的条件下, 是相对较好的单体浓度.
表 $1 \mathrm{Ru}\left(p\right.$-cymene) $\mathrm{HClPCy}_{3}$ 催化降冰片烯开环易位聚合溶剂篮选

Table 1 Screen of solvent for $\mathrm{Ru}(p$-cymene $) \mathrm{HClPCy}_{3}$ catalytic system ${ }^{a}$

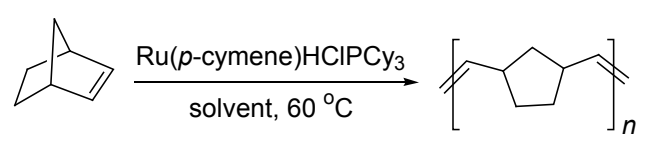

\begin{tabular}{ccc}
\hline Solvent & Conversion $/ \%$ & Trans $/ \%$ \\
\hline $\mathrm{CHCl}_{3}$ & 72.4 & 75 \\
$\mathrm{THF}$ & 87.9 & 88 \\
$\mathrm{PhCl}$ & 84.8 & 85 \\
\hline
\end{tabular}

${ }^{a}$ Conditions: $[\mathrm{M}]: 0.5 \mathrm{~mol} / \mathrm{L},[\mathrm{Ru}]: 2.5 \mathrm{mmol} / \mathrm{L}, 60{ }^{\circ} \mathrm{C}, 15 \mathrm{~min}$.

再高的浓度对于聚合反应转化率的提高基本没有影响. 接着, 我们考察了其它反应条件如单体与催化剂的比 例、反应温度和反应时间等参数(如 Supporting Information Figure S2, Figure S3 和 Figure S4 所示). 在 ROMP 聚合中，单体与催化剂的比例控制着聚合物高分子的分 子量, 同时也对转化率起到影响, 对照实验中(单体/催 化剂: 100/1，200/1，300/1，400/1，500/1，600/1，800/1, $1000 / 1$ ), 在 $100 / 1$ 到 600/1 的范围内, 转化率与其比例成 线性下降, 至 $800 / 1$ 和 $1000 / 1$ 时, 有明显降低. 在较低反 应温度下 $\left(30{ }^{\circ} \mathrm{C}, 40{ }^{\circ} \mathrm{C}\right)$ 聚合反应转化率不高, 在相对 提高的温度下 $\left(50{ }^{\circ} \mathrm{C}, 60{ }^{\circ} \mathrm{C}, 70{ }^{\circ} \mathrm{C}\right)$ 反应可以得到很好 的转化率.

因此, 通过上述的优化, 本文最终确定了 $\mathrm{Ru}\left(p\right.$-cymene)HCIPCy $\mathrm{H}_{3}$ 催化 ROMP 反应的条件: 单体浓 度: $0.5 \mathrm{~mol} / \mathrm{L} ; \mathrm{Ru}\left(p\right.$-cymene) $\mathrm{HClPCy}_{3}$ 浓度: $2.5 \mathrm{mmol} / \mathrm{L}$; 溶剂: $\mathrm{PhCl}$; 反应温度: $60{ }^{\circ} \mathrm{C}$; 反应时间: $15 \mathrm{~min}$. 在该 反应条件下，反应进行约 $2 \mathrm{~min}$ 时，反应液由于聚合物 浓度升高, 分子量增长, 粘度上升, 以至于出现了类似 凝胶的状态. 综上所述，验证了前面的假设，即用单核 $\mathrm{Ru}(p$-cymene $) \mathrm{HClPCy}_{3}$ 催化体系确实可以催化降冰片烯 的聚合反应.

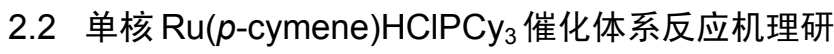
究

在已经得到验证的假设中 $\mathrm{Ru}(p$-cymene $) \mathrm{HClPCy}_{3}$ 之 所以存在催化活性是基于以下三个配体即：易解离的 $p$-cymene 配体，氢原子配体和稳定配合物的磷配体 $\mathrm{PCy}_{3}$, 但是, 在配合物 $\mathrm{Ru}(p$-cymene $) \mathrm{HClPCy}_{3}$ 中还存在 第四个配体, 即配合物中保留了的氯原子配体. 进一步 假设如果将这第四个配体换成氢原子配体，即去除氯原 子配体使整个配合物只保留负氢配体的特点, 是否还可 以保留聚合活性? 将上述四个特征组合, 可以得到结果 为配合物 $\mathrm{Ru}\left(p\right.$-cymene) $\mathrm{H}_{2} \mathrm{PCy}_{3}{ }^{[24]}$. 本文是通过 $\left[\mathrm{Ru}(p \text {-cymene }) \mathrm{Cl}_{2}\right]_{2}$ 合成了 $\mathrm{Ru}(p$-cymene $) \mathrm{Cl}_{2} \mathrm{PCy}_{3}$ 后, 再 在碱的条件下取代氯原子合成了 $\mathrm{Ru}(p$-cymene $) \mathrm{H}_{2} \mathrm{PCy}_{3}$. 将其作为催化剂进行降冰片烯的 ROMP 反应, 条件选 择 $\mathrm{Ru}(p$-cymene $) \mathrm{HClPCy}_{3}$ 催化体系的反应条件. 然而, 与 $\mathrm{Ru}(p$-cymene $) \mathrm{HClPCy}_{3}$ 呈现出的高催化性能相反的 是，在反应进行了相同时间甚至更长的反应时间如 $6 \mathrm{~h}$ 


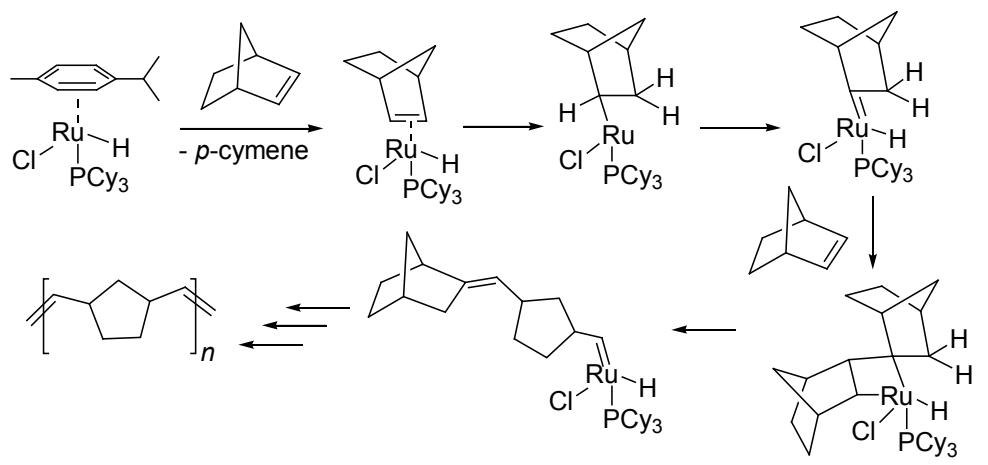

图 $2 \mathrm{Ru}\left(p\right.$-cymene) $\mathrm{HClPCy}_{3}$ 催化降冰片烯开环易位聚合的可能机理

Figure 2 Reaction mechanism proposed for $\mathrm{Ru}(p$-cymene $) \mathrm{HClPCy}_{3}$ catalytic system

后，降冰片烯的转化率仍然不足 30\% (Supporting information). 这一结果表明, 将单核钓配合物所具有的氯 原子配体取代成氢原子配体而得到的配合物 $\mathrm{Ru}(p$-cymene $) \mathrm{H}_{2} \mathrm{PCy}_{3}$ 没有如 $\mathrm{Ru}(p$-cymene $) \mathrm{HClPCy}_{3}$ 催化 降冰片烯 ROMP 反应的反应活性.

通过设计核磁监测反应，在 NMR 管中用 $0.5 \mathrm{~mL}$ $\mathrm{CDCl}_{3}$ 作为溶剂, 并加入了 $3 \mathrm{mg}$ 的 $\mathrm{Ru}(p$-cymene)$\mathrm{HClPCy}_{3}, 3.8 \mathrm{mg}$ 降冰片烯, 在 $60{ }^{\circ} \mathrm{C}$ 下反应 $40 \mathrm{~min}$, 从 ${ }^{1} \mathrm{H}$ NMR 图谱上, 我们观察到在 $\delta 9.64$ 处出现了一个很 小的单峰(Supporting Information Figure S5 所示), 其积 分面积大约是 $\mathrm{Ru}(p$-cymene $) \mathrm{HClPCy}_{3}$ 上与钓直接配位的 氢原子积分面积的 $6 \%$, 而在反应前并没有发现这个核 磁信号峰, 这个核磁峰很可能对应的是活性中心或催化 中间体.

根据上述的研究及本课题组在双核含负氢配体对 降冰片烯的开环易位聚合研究, 本文提出了如下的可能 的机理(如 Figure 2 所示): 催化中心钓配合物上的负氢 配体因为其较大的反式效应使较易解配位的配体更好 地解配位进而促进了配体 $p$-cymene 的解离, 从而使降 冰片烯更好地与钓中心配位提供了反应位点. 接着, 降 冰片烯的烯基插入 $\mathrm{Ru}-\mathrm{H}$ 键中形成烷基, 进而发生了 $\alpha$-消除其转变为 ROMP 活性增长点氢卡宾物质, 从而得 到高分子链 ${ }^{[22]}$.

\section{3 结论}

本文通过对含负氢配体的单核钓配合物 $\mathrm{Ru}$ (p-cymene) $\mathrm{HClPCy}_{3}$ 催化降冰片烯的 ROMP 反应的研 究, 验证了负氢配体对单核钓配合物对降冰片烯聚合反 应的作用，同时通过 $\mathrm{Ru}(p$-cymene $) \mathrm{H}_{2} \mathrm{PCy}_{3}$ 的聚合活性 研究进而验证了含有易解离的 $p$-cymene 配体、负氢配 体及磷配体 $\mathrm{PCy}_{3}$ 的单核钓配合物催化剂需同时含有氯 原子配体具有很好催化活性, 从而进一步验证了本课题
组在双核钓配合物催化降冰片烯研究中提出的含双氢 桥一氯桥的双核钓配合物为活性中心的假设.

\section{References}

[1] Sutthasupa, S.; Shiotsuki, M.; Sanda, F. Polym. J. 2010, 42, 905.

[2] Bielawski, C. W.; Grubbs, R. H. Prog. Polym. Sci. 2007, 32, 1.

[3] Black, G.; Maher, D.; Risse, W. In Handbook of Metathesis: Catalyst Development, Ed.: Grubbs, R. H., Wiley-VCH Verlag GmbH, 2008, pp. 2 71.

[4] Buchmeiser, M. R. Chem. Rev. 2000, 100, 1565.

[5] Schwab, P.; Grubbs, R. H.; Ziller, J. W. J. Am. Chem. Soc. 1996, 118 , 100.

[6] Schwab, P.; France, M. B.; Ziller, J. W.; Grubbs, R. H. Angew. Chem. Int. Ed. Engl. 1995, 34, 2039.

[7] Scholl, M.; Ding, S.; Lee, C. W.; Grubbs, R. H. Org. Lett. 1999, 1, 953.

[8] Harrity, J. P. A.; La, D. S.; Cefalo, D. R.; Visser, M. S.; Hoveyda, A. H. J. Am. Chem. Soc. 1998, 120, 2343.

[9] Garber, S. B.; Kingsbury, J. S.; Gray, B. L.; Hoveyda, A. H. J. Am. Chem. Soc. 2000, 122, 8168.

[10] Romulus, J.; Patel, S.; Weck, M. Macromolecules 2012, 45, 70.

[11] Park, H.; Choi, T.-L. J. Am. Chem. Soc. 2012, 134, 7270.

[12] Lai, W.-Y.; Balfour, M. N.; Levell, J. W.; Bansal, A. K.; Burn, P. L.; Lo, S.-C.; Samuel, I. D. W. Macromolecules 2012, 45, 2963.

[13] Keitz, B. K.; Fedorov, A.; Grubbs, R. H. J. Am. Chem. Soc. 2012, 134, 2040.

[14] Qian, Y.; Chen, B.; Jin, J.; Huang, J. Acta Chim. Sinica 2000, 58, 1050. (钱延龙, 陈斌, 金军挺, 黄吉玲, 化学学报, 2000, 58 , 1050.)

[15] Demonceau, A.; Stumpf, A. W.; Saive, E.; Noels, A. F. Macromolecules 1997, 30, 3127.

[16] Delaude, L.; Demonceau, A.; Noels, A. F. Macromolecules 1999, 32, 2091.

[17] Jan, D.; Delaude, L.; Simal, F.; Demonceau, A.; Noels, A. F. J. Organomet. Chem. 2000, 606, 55 .

[18] Furstner, A.; Ackermann, L. Chem. Commun. 1999, 95.

[19] Delaude, L.; Demonceau, A.; Noels, A. F. Chem. Commun. 2001, 986.

[20] Louie, J.; Grubbs, R. H. Angew. Chem. Int. Ed. 2001, 40, 247.

[21] Delaude, L.; Szypa, M.; Demonceau, A.; Noels, A. F. Adv. Synth. Catal. 2002, 344, 749.

[22] Chen, J.; Chen, X.; Zhu, C.; Zhu, J. J. Mol. Catal. A-Chem. 2014, 394, 198.

[23] Solari, E.; Gauthier, S.; Scopelliti, R.; Severin, K. Organometallics 2009, 28, 4519

[24] Demerseman, B.; Mbaye, M. D.; Sémeril, D.; Toupet, L.; Bruneau, C.; Dixneuf, P. H. Eur. J. Inorg. Chem. 2006, 2006, 1174. 